\title{
Hybrid surgery for an arteriovenous malformation fed by an accessory middle cerebral artery and drained by a developmental venous anomaly: A case report and literature review
}

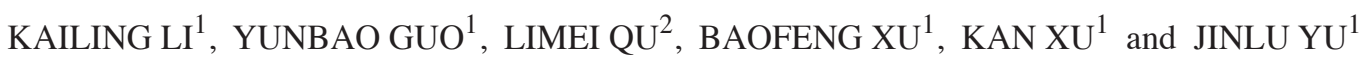 \\ Departments of ${ }^{1}$ Neurosurgery and ${ }^{2}$ Pathology, The First Hospital of Jilin University, Changchun, Jilin 130021, P.R. China
}

Received January 2, 2018; Accepted June 21, 2018

DOI: $10.3892 /$ etm.2018.6372

\begin{abstract}
An accessory middle cerebral artery (AMCA), which mainly acts in the collateral circulation of the middle cerebral artery (MCA), is a rare anatomic malformation. Similar to other intracranial vessels, cerebrovascular disease can occur in the AMCA. However, the development of an arteriovenous malformation (AVM) in the AMCA is very rare, especially in conjuction with developmental venous anomalies (DVAs). Here, a rare case of an AMCA combined with an AVM and a DVA was reported. The patient was a 47-year-old female with intracranial hemorrhage at symptom onset. CT and MRI showed lesions in the left Sylvian fissure and insula accompanied by hemorrhage. DSA suggested a left AMCA; an AVM of the AMCA was located in the deep Sylvian fissure. The AVM was diffusely developed and drained into the DVA. The operation was performed in a hybrid operating room. The major feeding artery of the AVM, which was derived from the AMCA, was clipped, then the AVM and DVA were subsequently removed. Intraoperative DSA showed that the AVM and DVA were radically removed. A pathological examination confirmed the presence of an AVM. The patient recovered well and was discharged. Therefore, as highlighted in this case report, rare AVMs can be found in AMCAs and can even occur simultaneously with a DVA. Hybrid surgical treatment can be used to remove AVMs and can lead to an improved prognosis.
\end{abstract}

\section{Introduction}

An accessory middle cerebral artery (AMCA) is a relatively uncommon anomaly of the middle cerebral artery (MCA) in which the AMCA arises from the anterior cerebral artery (ACA) that coexists with the main trunk of the MCA $(1,2)$. The angiographic incidence of AMCA is $0.32 \%$ (3). Anatomically,

Correspondence to: Professor Jinlu Yu, Department of Neurosurgery, The First Hospital of Jilin University, 71 Xinmin Avenue, Changchun, Jilin 130021, P.R. China

E-mail: jinluyu@hotmail.com

Key words: accessory middle cerebral artery, arteriovenous malformation, developmental venous anomaly, hybrid surgery an AMCA mainly acts as collateral circulation for the MCA. Similar to other intracranial vessels, the AMCA is involved in many diseases, such as aneurysms and cerebral infarction (4-6). Although arteriovenous malformations (AVMs) can theoretically occur in an AMCA, this finding is extremely rare (7). Most AVMs drain through the veins of the brain, and the drainage vein has, in some patients, been found to be enlarged or stenosed or to exhibit tortuousness (8). However, AVMs accompanied by developmental venous anomalies (DVAs) are rare, and only approximately 20 relevant articles have been published in the literature to date (7). A DVA is a benign and mostly silent condition without clinical symptoms (9). However, when a DVA merges with an AVM, due to the high blood flow in the AVM, drainage of blood from the DVA is difficult, resulting in drainage resistance. This feature creates complications within the AVM and makes DVAs prone to bleeding (10). Cases in which an AMCA was accompanied by an AVM and a DVA are rarely reported. However, the treatment of one such case is reported in this paper, and the relevant literature is reviewed.

\section{Case report}

A 47-year-old woman was admitted due to a headache accompanied by nausea and vomiting for $1 \mathrm{~h}$. The patient had always been healthy without any history of hypertension or diabetes. This disease was not induced by any other condition. The patient developed a sudden and severe headache at rest and subsequently developed nausea and vomiting accompanied by the inability to use her right limb and dysphasia. The patient was admitted to our hospital after symptom onset. At the time of presentation, the patient was alert with no seizure. An admission examination showed the following. The patient was alert but exhibited incomplete motor aphasia. The left limbs moved freely, while the right limbs were reflexive (muscle strength grade III). The Babinski sign was positive in the right lower limb. A head CT and MRI after admission showed lesions in the left Sylvian fissure and insular lobe. CT showed high and slightly high mixed densities. The signals were confounding on MRI. An abnormally thickened vein extended from the brain surface to the deep lesions (Fig. 1). Further DSA revealed a vascular malformation in the left MCA and an AMCA that originated from the anterior communicating artery (Fig. 2A and B). The vascular malformation in the 
AMCA was seen in the deep Sylvian fissure (Fig. 2C and D). The vascular malformation was diffuse, and a small number of fine branching arteries served as its blood supply. It drained into the DVA (Fig. 3). The patient was diagnosed with a left frontal hemorrhage with an AMCA that merged with an AVM and a DVA. AVM resection was scheduled to be performed in a hybrid operating room.

Surgical treatment was performed in a hybrid operating room. Femoral artery puncture was performed, and an arterial sheath was inserted. A left expanded pterional approach was adopted during surgery. The AMCA originating from the anterior communicating artery could be seen at the chiasmatic cistern after the Sylvian fissure on the left side was opened (Fig. 4A). The AVM was located on the surface of the insular lobe of the deep Sylvian fissure. The major feeding artery of the AVM, which originated from the AMCA, was clipped. The AVM and the DVA were subsequently removed from the AVM, which resulted in obsolete hemorrhage in the AVM. The AVM was diffuse, and the venous end drained into the DVA (Fig. 4B). The final pathological examination after removal showed an irregular vascular shape that consisted of various types of expanded and transparent veins and abnormal muscularized arteries. An abnormal arteriovenous anastomosis was also observed along with brain tissues between vessels and was diagnosed as an AVM (Fig. 4C and D). DSA was performed after AVM resection, which showed that the AVM and DVA were completely removed (Fig. 5). The patient recovered well within one week of the surgery. A one-month follow-up CT scan showed good recovery in the brain tissue (Fig. 6). The patient was in good condition, had grade IV muscle strength in the right limb, and could take care of herself.

\section{Discussion}

In 1973, Teal et al (11) proposed using the term 'AMCA' to describe an anomalous artery arising from the ACA. The origin of an AMCA can be the proximal or distal ACA, and the vessel that arises from the ACA then runs in the Sylvian fissure along with the main trunk of the MCA to supply part of the MCA territory $(11,12)$. The angiographic incidence of AMCA is $0.32 \%$ (3). Manelfe et al (13) classified AMCA into three types: Type 1 is an anomalous vessel that arises from the internal carotid artery at a point proximal to its bifurcation (the duplicated MCA in Teal's classification), type 2 originates from the proximal portion of the ACA, and type 3 originates from the distal portion of the first segment of the ACA (A1) near the anterior communicating artery (14). The AMCA in this patient originated from the anterior communicating artery and was classified as Manelfe type 3. Typically, an AMCA serves as the collateral blood supply to the MCA territory. However, the clinical significance of AMCAs remains obscure (15).

Similar to normal MCAs, AMCAs can also be associated with many diseases, such as intracranial aneurysms (4), cerebral infarction (5), and moyamoya disease (6). However, an AMCA combined with an AVM is very rare, especially with the vein of the AVM draining into the DVA, as in this case. Due to differences in the development of the AMCA, such arteries can vary in diameter (16), and there are large differences in the feeding area distribution of its arteries (17).
For example, when the blood supply to the AMCA is extensive, the consequences are more severe if the AMCA undergoes an acute infarction $(5,18)$. Moreover, when the ACMA is large with abundant blood flow, the artery is also prone to aneurysms $(19,20)$. Therefore, the development of an AMCA may be associated with diseases. Although an AVM combined with an AMCA is relatively rare, we report such a case in this article.

AVMs are a type of congenital cerebrovascular condition which, along with capillary telangiectasia, cavernous malformation, and DVA, represent the 4 most commonly recognized subgroups of cerebrovascular malformations (21). An AVM is a collection of arteries and veins that form without an intervening capillary bed (22). However, a DVA is usually a collection of radiating veins that converge on a large and centrally located draining vein (23). This is classically described as having a fan-shaped 'caput medusae' appearance, according to a large prospective autopsy series, DVA is the most common type of vascular malformation in the brain. It is now accepted that DVA generally follow a benign clinical course (24).

Therefore, during embryonic development, an AVM and a DVA may occur on an AMCA, as is the case in the patient described in this paper. It is possible that the AVM formed first, and then venous hypertension around the draining vein occurred, and the disturbed normal venous drainage due to venous hypertension caused by preexisting arteriovenous shunting may have promoted the development of a DVA (25). The first patient with a coexisting AVM and DVA was reported by Huang et al (26) in 1984, and a literature review of 22 cases was published by Zhang et al (7) in 2017. However, no reports have described an AVM accompanied by both a DVA and an AMCA.

When an AVM is accompanied by a DVA, the risk of hemorrhage is as high as $68 \%$, and these patients are more prone to bleeding than are those with a single AVM (38\%) (7). This finding may be related to the tenuous and slow-adapting terminal angioarchitecture that could lead to suboptimal venous drainage in the DVA $(27,28)$. Therefore, patients with both an AVM and a DVA must be treated when rupture and bleeding occur. Microsurgical resection is an effective method for treating a ruptured AVM, especially when the AVM is shallow and is located in a non-relocating area (29). Whether the DVA needs to be removed during AVM resection must be determined. Despite the risk imposed by the aberrant drainage, successful neurosurgical management of these transitional lesions should target the AVM and retain a tissue-dependent DVA with low hemorrhagic risk. This prevents the DVA from draining normal brain tissue after removal and prevents catastrophic venous infarction (7).

The patient described in our paper had a diffusely developed AVM with unclear boundaries. We found bulky veins in the DVA, and the AVM around the DVA was gradually removed. Intraoperative DSA showed that the AVM was completely removed, and the DVA was not visible. We adopted a hybrid operation procedure for this patient because it ensured the complete clearance of the AVM. Intraoperative DSA can also be performed to accurately determine the position of the AVM $(30,31)$. No serious complications occurred after the operation, and the patient's physical activity gradually recovered. However, a one-month postoperative follow-up CT 

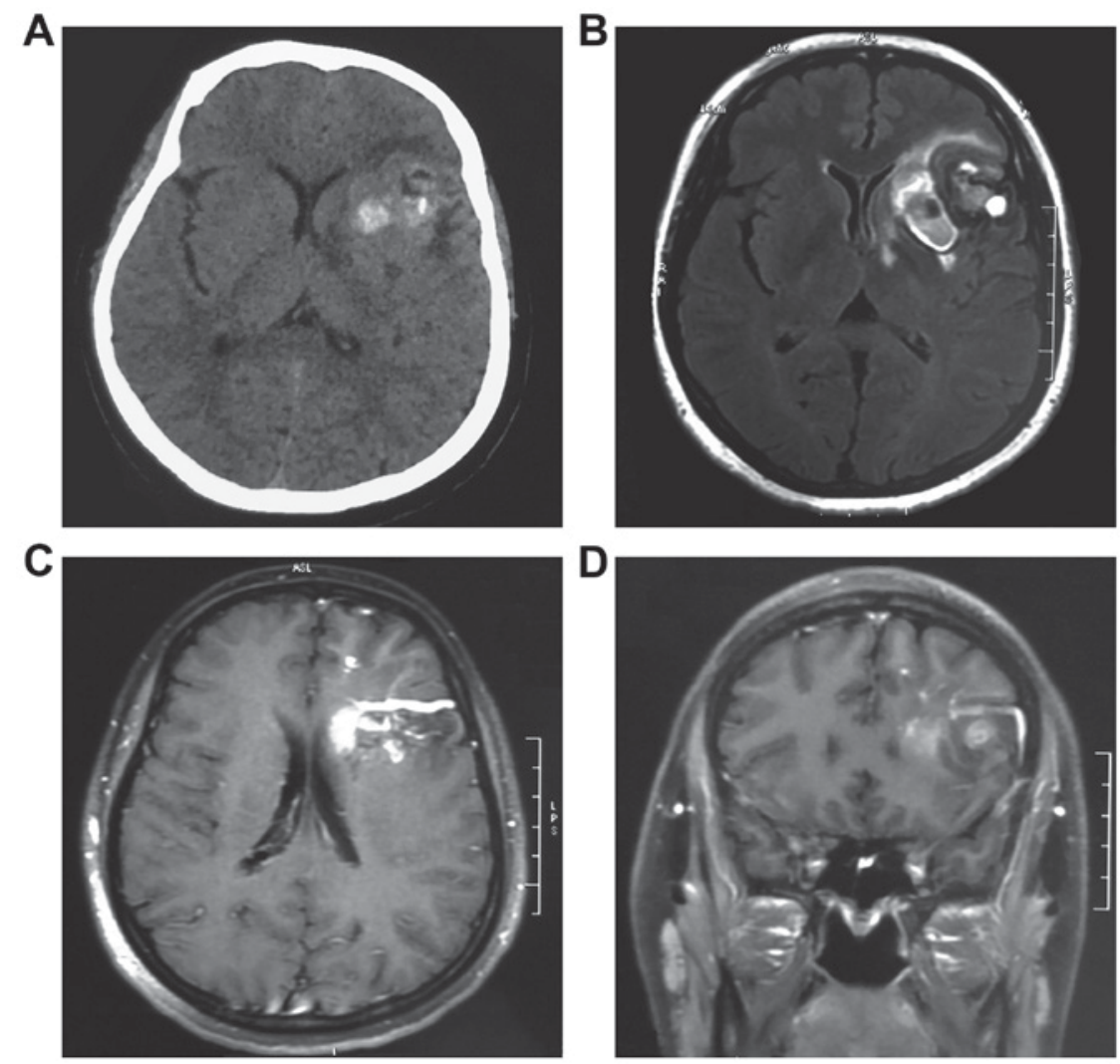

Figure 1. Preoperative CT and MRI images. (A) CT scan showing lesions in the deep left Sylvian fissure and the insular lobe with uneven, high and slightly high mixed density. (B) A flair MR image showing a confounding signal located in the deep Sylvian fissure that involved the insular lobe and part of the island surface. (C) Axial and (D) coronal MRI-enhanced T1 WI images showing an abnormally thick vein running from the surface of the brain to the deep lesion.
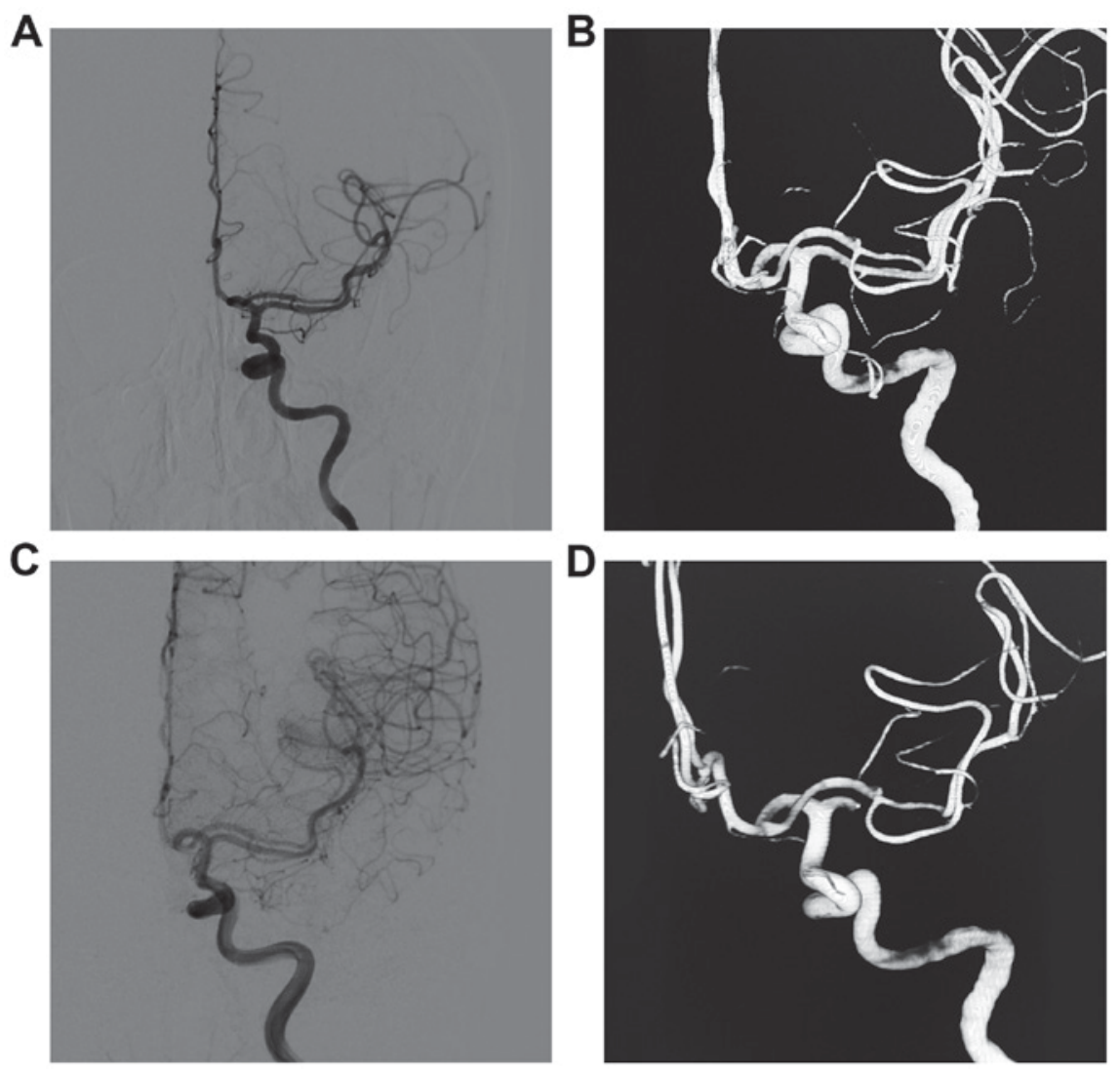

Figure 2. Preoperative DSA image. (A) 2D- and (B) 3D-reconstructed DSA images showing a vascular malformation in the middle cerebral artery along with an accessory middle cerebral artery that originated from the anterior communicating artery. (C) Vascular variations in the accessory middle cerebral artery. (D) The normal MCA was cut to locate the AMCA vascular malformation. MCA, middle cerebral artery; AMCA, accessory middle cerebral artery. 

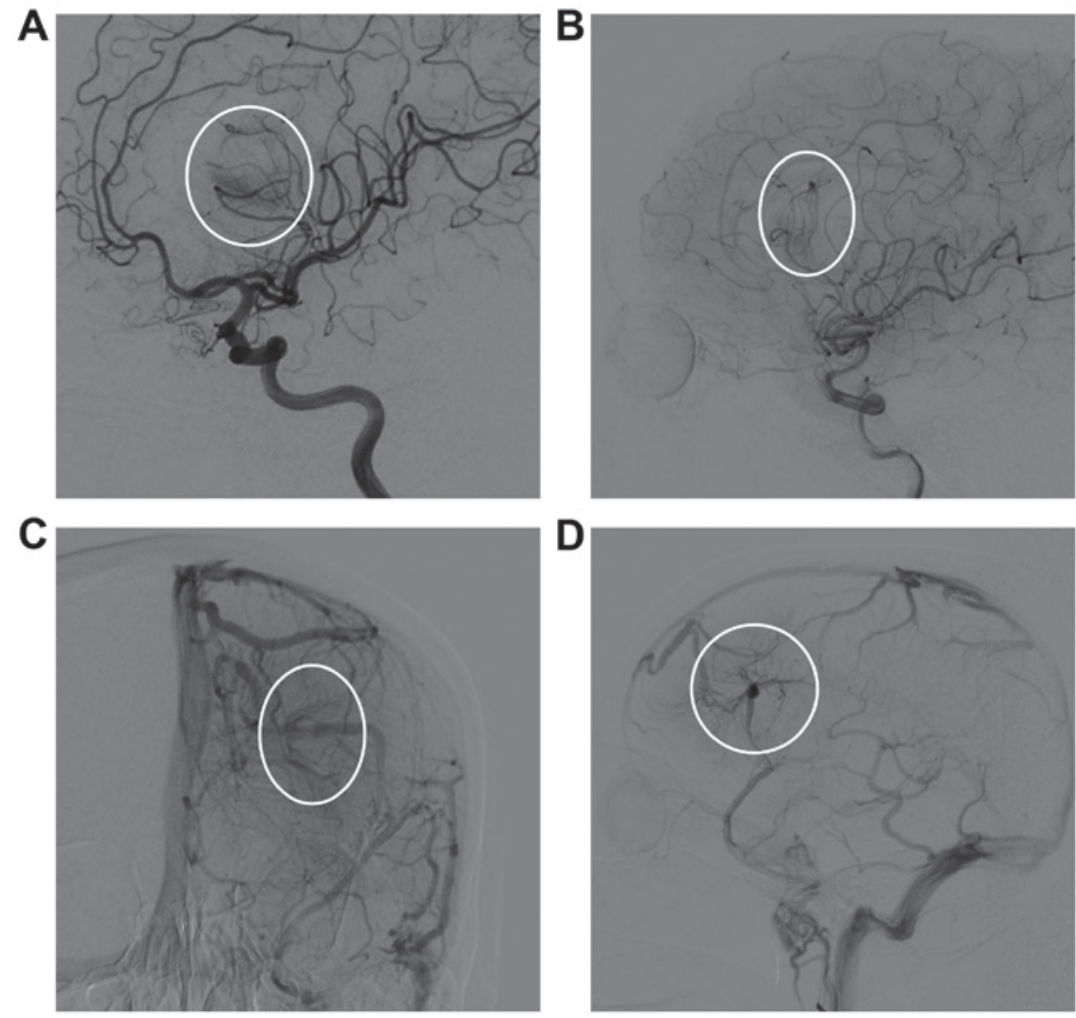

Figure 3. Details of the vascular malformation. (A and B) DSA in the arterial phase showing that the vascular malformation originated from the insular lobe of the accessory middle cerebral artery. The vascular malformation was diffuse. The artery serving as the blood supply was very small (see circle) and flowed into the DVA. (C and D) The DVA in the venous phase exhibited a DVA caput medusae (see circle). The vascular malformation accorded with the imaging for the AVM. DVAs, developmental venous anomalies; AVM, arteriovenous malformation.
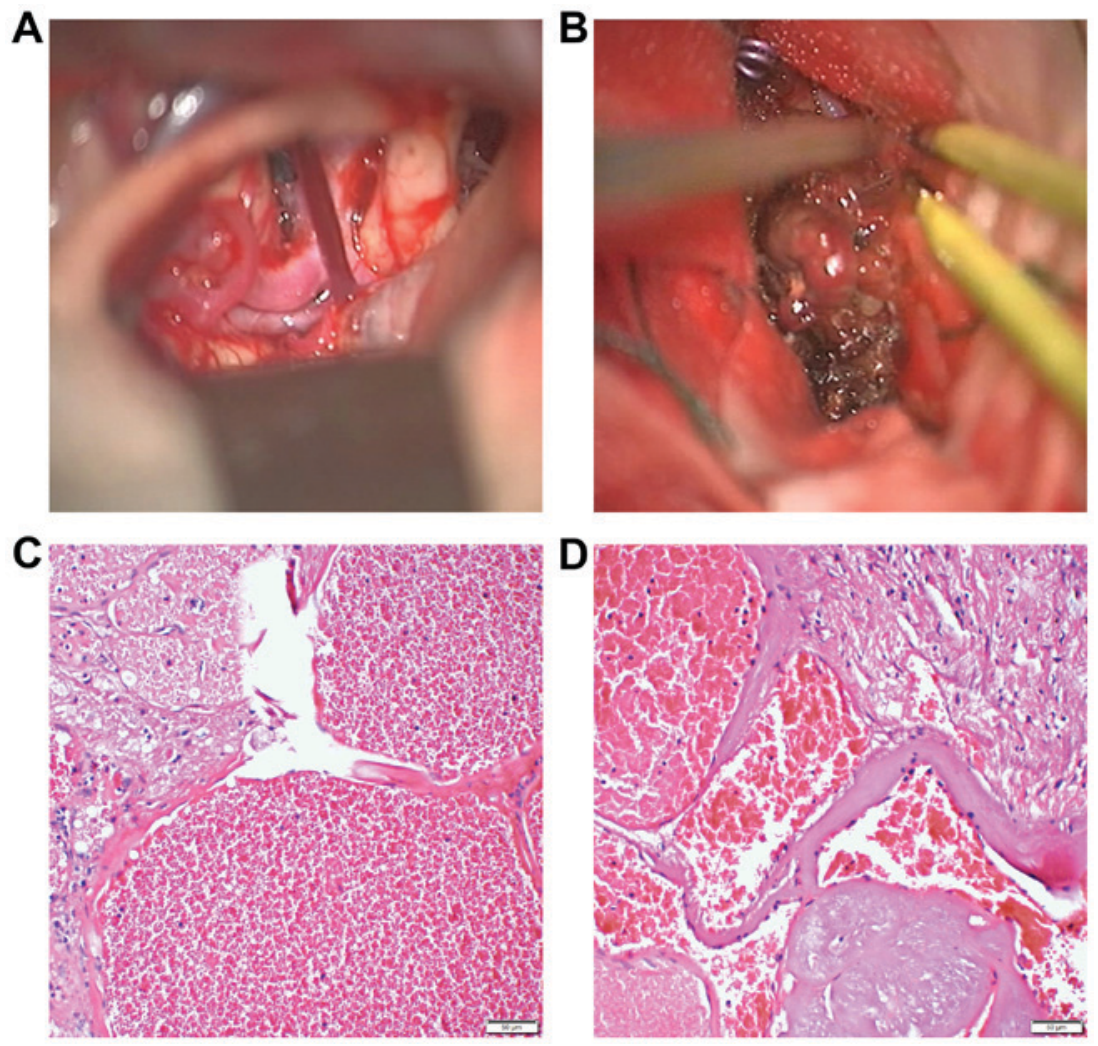

Figure 4. Intraoperative imaging and pathological images. (A) The AMCA originating from the anterior communicating artery could be seen at the left of the chiasmatic cistern. (B) The AVM was located on the deep Sylvian fissure. (C and D) H\&E staining (magnification, x200) showed blood vessels with an irregular shape and size. Lesions consisting of various types of expanded and transparent veins and abnormal muscularized arteries were visible. Vascular structures of single fiber tissue, which are difficult to distinguish, as well as abnormal arteriovenous anastomosis, were visible. The brain tissue was visible among the vessels. AMCA, accessory middle cerebral artery; AVM, arteriovenous malformation. 

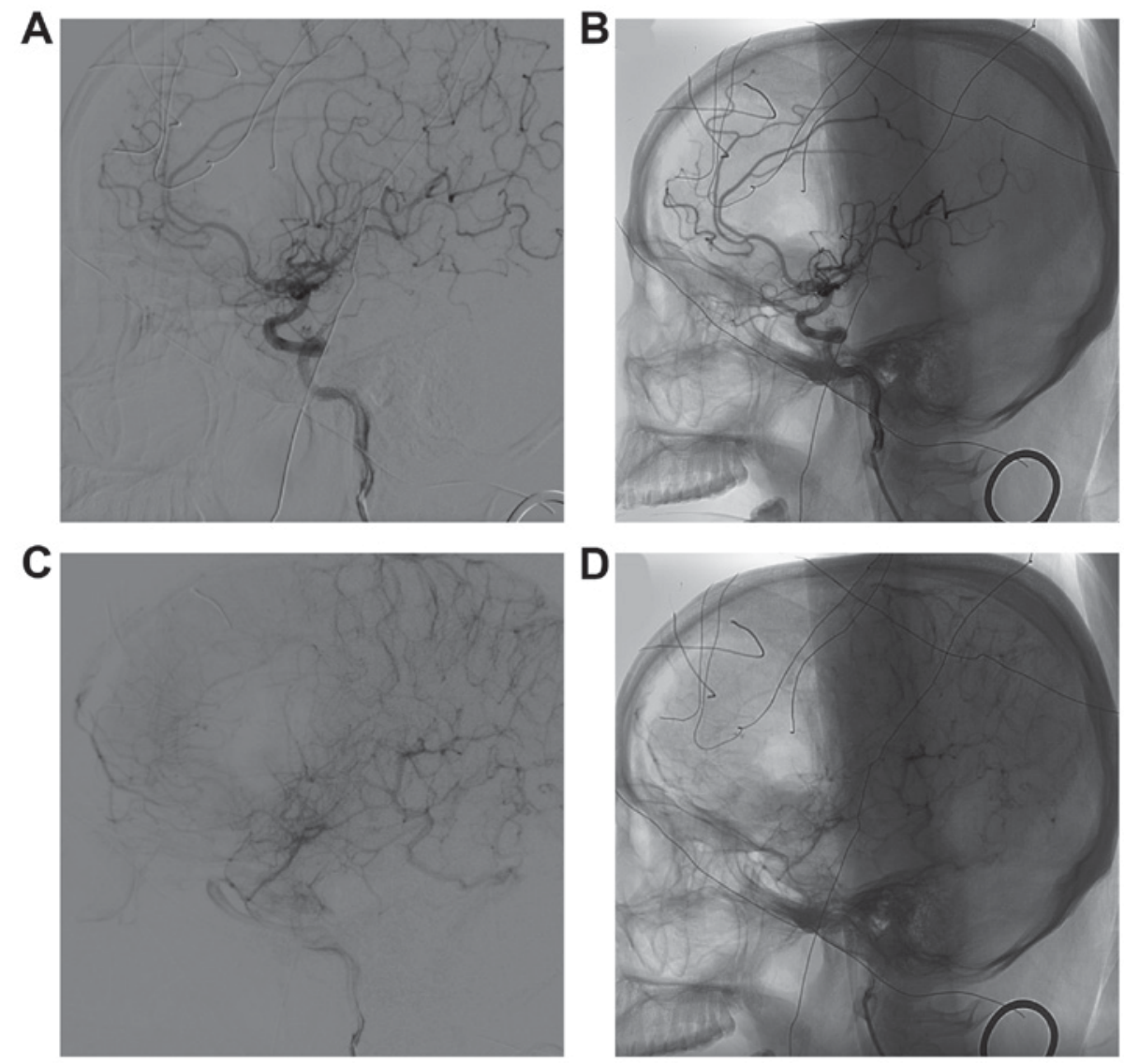

Figure 5. Postoperative DSA images. Postoperative DSA showing complete removal of the AVM. The AVM and drainage veins were not visible. (A) Early artery phase. (B) Early artery phase with mask image. (C) Venous phase. (D) Venous phase with mask image. AVM, arteriovenous malformation.
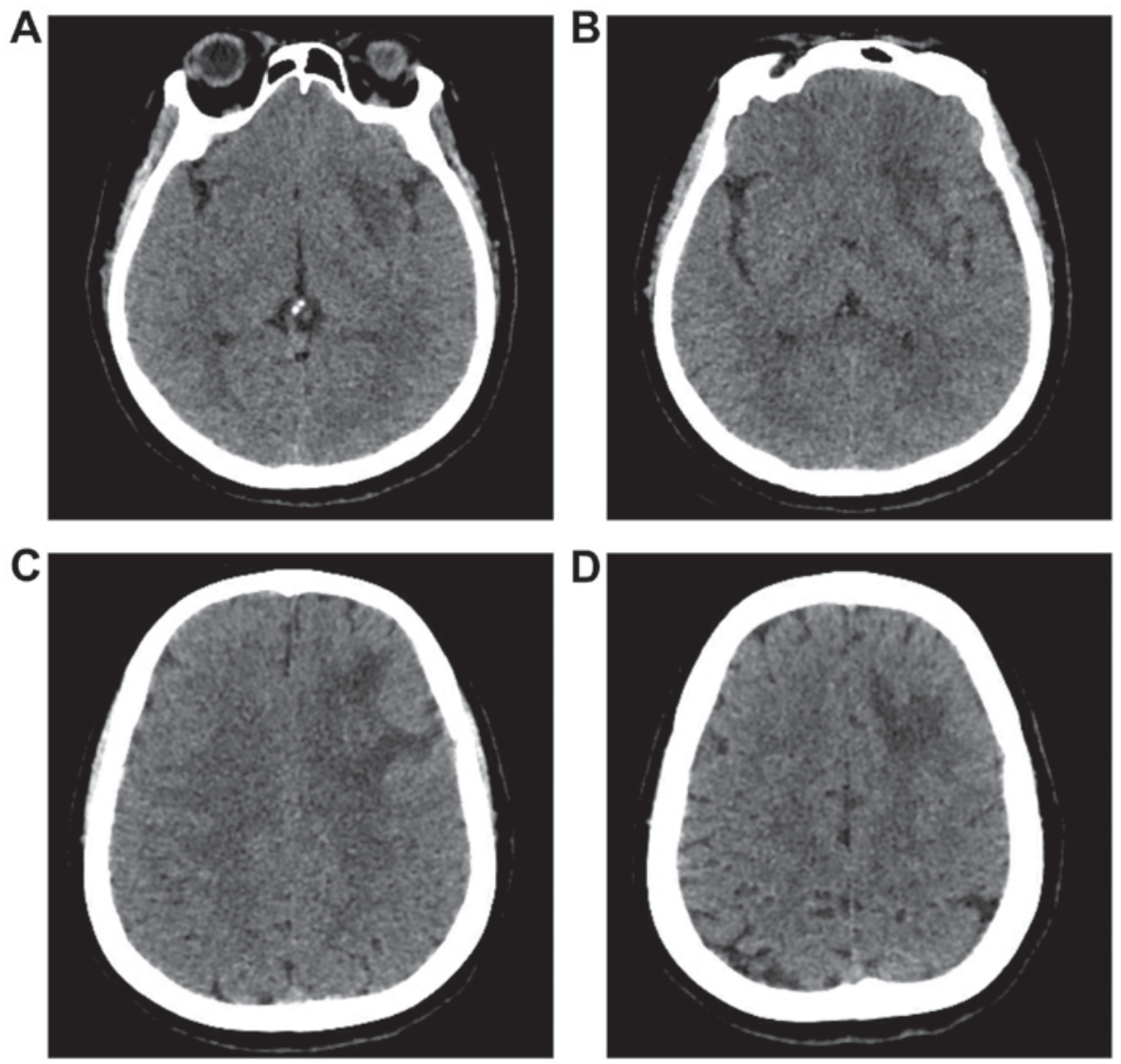

Figure 6. CT images at 6 months postoperative. (A-D) Postoperative CT images showing that the brain tissue had recovered, with mild edema. 
scan showed that cerebral edema persisted, and this may have be related to the removal of the DVA during the operation.

In addition to microsurgical resection of AVMs and DVAs, radiosurgery, embolization and combination therapy are effective treatments (7). In 2017, Zhang et al (7) reviewed 22 cases of coexisting AVMs and DVAs. Of these, stereotactic radiosurgery was performed in 7 cases, embolization was performed in 6 cases, surgical resection was performed in 4 cases, and multimodal therapy was performed in 5 cases. AVMs were treated by targeted embolization, and the DVAs were carefully preserved (32). When an AVM is accompanied by a DVA and no evidence of bleeding is present, radiotherapy is also an effective treatment. Aksoy et al (10) performed radiosurgery in a patient with both an AVM and a DVA and reported a good patient outcome at the 6-month follow-up evaluation. However, as the effect of radiation is generally delayed until 2-4 years, whether the patient remained well at a longer-term follow-up is unclear (10). Nevertheless, it is important to note that only the AVM should be obliterated by radiosurgery, whereas the DVA should be completely preserved (9).

Therefore, as reported in this rare case, AVMs can be found in an AMCA and can also be simultaneously observed with a DVA. Hybrid surgical treatment can be used to remove AVMs, and this approach can improve the patient's prognosis.

\section{Acknowledgements}

Not applicable.

\section{Funding}

No funding was received.

\section{Availability of data and materials}

The datasets used and/or analyzed during the current study are available from the corresponding author on reasonable request.

\section{Authors' contributions}

KL analysed the patient data and wrote the initial draft. YG, LQ and BX collected the images of the patients and analyzed the literature. JY and KX were the surgeons who treated the patient. All authors read and approved the final manuscript.

\section{Ethics approval and consent to participate}

The present study was approved by the Ethics Committee of the First Hospital of Jilin University and written informed consent form was obtained from the patients. All procedures performed were in accordance with the ethical standards of the institutional and/or national research committee and with the 1964 Helsinki declaration and its later amendments or comparable ethical standards.

\section{Patient consent for publication}

Not applicable.

\section{Competing interests}

The authors have declared that they have no competing interests.

\section{References}

1. Fujiwara K, Saito K and Ebina T: Saccular aneurysm of the accessory middle cerebral artery-case report. Neurol Med Chir (Tokyo) 43: 31-34, 2003.

2. Uchiyama N: Anomalies of the middle cerebral artery. Neurol Med Chir (Tokyo) 57: 261-266, 2017.

3. Watanabe T and Togo M: Accessory middle cerebral artery. Report of four cases. J Neurosurg 41: 248-251, 1974.

4. Lee CC, Liu ZH, Jung SM and Yang TC: Ruptured aneurysm of the accessory middle cerebral artery associated with moyamoya disease: A case report. Chang Gung Med J 34: 541-547, 2011.

5. Hiramatsu Y, Wakita M, Matsuoka H, Kasuya J, Hamada R and Takashima H: Cerebral infarction associated with accessory middle cerebral arteries: Two case reports. Intern Med 53: 1381-1384, 2014.

6. Komiyama $\mathrm{M}$ and Yasui T: Accessory middle cerebral artery and moyamoya disease. J Neurol Neurosurg Psychiatry 71: 129-130, 2001.

7. Zhang M, Connolly ID, Teo MK, Yang G, Dodd R, Marks M, Zuccarello M and Steinberg GK: Management of arteriovenous malformations associated with developmental venous anomalies: A literature review and report of 2 cases. World Neurosurg 106: 563-569, 2017.

8. Kurita H, Shin M, Ueki K, Kawamoto S and Kirino T: Congestive brain oedema associated with a pial arteriovenous malformation with impaired venous drainage. Acta Neurochir (Wien) 143: 339-342, 2001.

9. Kurita H, Sasaki T, Tago M, Kaneko Y and Kirino T: Successful radiosurgical treatment of arteriovenous malformation accompanied by venous malformation. AJNR Am J Neuroradiol 20: 482-485, 1999.

10. Aksoy FG, Gomori JM and Tuchner Z: Association of intracerebral venous angioma and true arteriovenous malformation: A rare, distinct entity. Neuroradiology 42: 455-457, 2000.

11. Teal JS, Rumbaugh CL, Bergeron RT and Segall HD: Anomalies of the middle cerebral artery: Accessory artery, duplication and early bifurcation. Am J Roentgenol Radium Ther Nucl Med 118: 567-575, 1973.

12. Komiyama M, Nakajima H, Nishikawa M and Yasui T: Middle cerebral artery variations: Duplicated and accessory arteries. AJNR Am J Neuroradiol 19: 45-49, 1998.

13. Manelfe C, David J and Rascol A: L'artère cérébrale moyenne accessoire. A propos de 17 cas. Société Française de Neuroradiologie, Paris, 1975 (In French).

14. Abanou A, Lasjaunias P, Manelfe C and Lopez-Ibor L: The accessory middle cerebral artery (AMCA). Diagnostic and therapeutic consequences. Anatom Clin 6: 305-309, 1984.

15. Komiyama M, Nishikawa $M$ and Yasui T: The accessory middle cerebral artery as a collateral blood supply. AJNR Am J Neuroradiol 18: 587-590, 1997.

16. Reis CV,Zabramski JM, Safavi-Abbasi S, Hanel RA, Deshmukh P and Preul MC: The accessory middle cerebral artery: Anatomic report. Neurosurgery 63 (1 Suppl 1): ONS10-ONS14, 2008.

17. Takahashi M, Uchino A and Suzuki C: Anastomosis between accessory middle cerebral artery and middle cerebral artery diagnosed by magnetic resonance angiography. Surg Radiol Anat 39: 685-687, 2017.

18. Liu ZS, Zhou LJ, Sun Y, Kuang XW, Wang W and Li C: Sufficient collateral blood supply from accessory middle cerebral artery in a patient with acute ischemic stroke. Interv Neuroradiol 21: 215-217, 2015.

19. Teramoto S, Tokugawa J, Nakao Y and Yamamoto T: Caudate haemorrhage caused by pseudoaneurysm of accessory middle cerebral artery. BMJ Case Rep 2015: pii: bcr2015213335, 2015.

20. Parthasarathy R, Goel G, Gupta V, Narang KS, Anand S and Jha AN: Endovascular glue embolization of dissecting aneurysm of type-3 accessory middle cerebral artery: A contralateral approach. Interv Neuroradiol 21: 664-668, 2015.

21. McCormick WF: The pathology of vascular 'arteriovenous' malformations. J Neurosurg 24: 807-816, 1966.

22. Chen W, Choi EJ, McDougall CM and Su H: Brain arteriovenous malformation modeling, pathogenesis, and novel therapeutic targets. Transl Stroke Res 5: 316-329, 2014. 
23. Brinjikji W, El-Masri AE, Wald JT, Flemming KD and Lanzino G: Prevalence of cerebral cavernous malformations associated with developmental venous anomalies increases with age. Childs Nerv Syst 33: 1539-1543, 2017.

24. Sarwar M and McCormick WF: Intracerebral venous angioma. Case report and review. Arch Neurol 35: 323-325, 1978.

25. Yanaka K, Hyodo A and Nose T: Venous malformation serving as the draining vein of an adjoining arteriovenous malformation. Case report and review of the literature. Surg Neurol 56: 170-174, 2001.

26. Huang Y, Robbins A, Patel S and Chaudhary M: Cerebral venous malformations and a new classification of cerebral malformations. The cerebdral venous system and its disorders 373-474, 1984.

27. da Costa L, Wallace MC, Ter Brugge KG, O'Kelly C, Willinsky RA and Tymianski M: The natural history and predictive features of hemorrhage from brain arteriovenous malformations. Stroke 40: 100-105, 2009.

28. Shakur SF, Liesse K, Amin-Hanjani S, Carlson AP, Aletich VA, Charbel FT and Alaraj A: Relationship of cerebral arteriovenous malformation hemodynamics to clinical presentation, angioarchitectural features and hemorrhage. Neurosurgery 63 (Suppl 1): S136-S140, 2016.
29. Magro E, Gentric JC, Batista AL, Kotowski M, Chaalala C, Roberge D, Weill A, Stapf C, Roy D, Bojanowski MW, et al: The treatment of brain AVMs Study (TOBAS): An all-inclusive framework to integrate clinical care and research. J Neurosurg 128: $1823-1829,2018$

30. Shi L, Li W, Xu K, Guo Y and Yu J: Current status of combined surgical and endovascular methods for intracranial neurovascular diseases in a hybrid operating room. Int J Clin Exp Med 9: 20741-20753, 2016

31. Yu J, Guo Y, Xu B, Chen X and Xu K: Onyx embolization and surgical removal as a treatment for hemorrhagic AVM in a hybrid operating room. Int J Clin Exp Med 9: 22494-22501, 2016.

32. Fok KF, Holmin S, Alvarez H, Ozanne A, Krings $\mathrm{T}$ and Lasjaunias PL: Spontaneous intracerebral hemorrhage caused by an unusual association of developmental venous anomaly and arteriovenous malformation. Interv Neuroradiol 12: 113-121, 2006.

This work is licensed under a Creative Commons Attribution-NonCommercial-NoDerivatives 4.0 International (CC BY-NC-ND 4.0) License. 